\title{
AN INVESTIGATION INTO ELECTROMAGNETIC BASED IMPEDANCE TOMOGRAPHY USING REALISTIC HUMAN HEAD MODEL
}

\author{
AWAIS MUNAWAR ${ }^{1}$, ZARTASHA MUSTANSAR ${ }^{1}$, AHMED E NADEEM¹, MAHMOOD AKHTAR² \\ ${ }^{1}$ Research Center for Modeling and Simulation, National University of Sciences and Technology, Islamabad, Pakistan, ${ }^{2}$ College of Electrical \\ and Mechanical Engineering, National University of Sciences and Technology, Islamabad, Pakistan \\ Email: awaismunawar.phd06@rcms.nust.edu.pk \\ Received: 25 Apr 2016 Revised and Accepted: 27 May 2016
}

\begin{abstract}
The objective of this research is to investigate the feasibility of Electromagnetic based Impedance Tomography (EMIT) for brain stroke detection, localization and classification. Electromagnetic based Impedance Tomography employing microwave imaging technique is an emerging brain stroke diagnostic modality. It relies on the significant contrast between dielectric properties of the normal and abnormal brain tissues. To study the interaction between micro-wave signals and head tissues, the simulations are performed using a geometrically simple 3-D ellipsoid head model with emulated stroke. Finite Element numerical technique is adopted to find the solution of Maxwell's equations to measure the transmitted and backscattered signals in forward problem. Contrast Source Inversion technique is proposed to solve the inverse scattering problem and reconstruct brain images based on calculated dielectric profiles. Detailed analysis is performed to determine the safety limits of transmitted signals to minimize ionizing effects while ensuring maximum penetration. The simulations verify the inhomogeneous and frequency-dispersive behavior of brain tissue's dielectric properties. The solution of the forward problem demonstrates the microwave signals scattering by the multilayer structure of the head model, duly validated by analytical results. The scattering phenomena can be fully capitalized by image reconstruction algorithm to obtain brain images and detect stroke presence. The initial results obtained in this research and prior work indicates that EMIT-based head imaging system has a potential for rapid stroke detection, classification, and continuous brain monitoring and offers a comparatively cost-effective solution.
\end{abstract}

Keywords: Brain Stroke, EMIT, MWT, Forward Problem, FEM, Inverse Problem, CSI

(c) 2016 The Authors. Published by Innovare Academic Sciences Pvt Ltd. This is an open access article under the CC BY license (http://creativecommons.org/licenses/by/4.0/) DOI: http://dx.doi.org/10.22159/ijpps.2016v8s2.15217.

\section{INTRODUCTION}

A stroke is an interruption in the blood supply to brain tissues which causes a denial of oxygen and nutrients, thereby resulting in rapid loss of brain functions and often death. Brain stroke and traumatic brain injuries (TBIs) are ranked as the third major cause of death in the world after heart and cancer diseases. In this study, two major types of brain stroke are considered; hemorrhagic stroke and ischemic stroke [1]. The hemorrhagic stroke is caused by the burst of the blood vessel, whereas ischemic stroke is caused by the blockage of blood arteries and intracranial bleeding is the most common form of TBIs [2, 3]. Both types of stroke and TBIs have some common symptoms such as; dizziness, slurred speech, difficulty swallowing and sudden numbness of body parts. However, the course of treatment for each type is quite different. A decision needs to be taken by the doctor typically within 3-4 h from the onset of stroke symptoms [4]. This leads to establish the urgency to detect the type of stroke reliably. This section will provide a rationale about the techniques used for stroke detection in the literature to-date. A brief description of EMIT technique including forward problem solution, frequency dispersion in brain tissues and their dielectric properties are discussed at the end.

In order to ensure an accurate treatment for the effective recovery, the decision relies on the fast detection as well as localization of stroke. To achieve this, certain diagnostic techniques are in practice that includes both the non-visual and imaging modalities. The nonvisual modality uses methods like Electroencephalography (EEG), Magnetoencephalography (MEG), Electrical Impedance Tomography (EIT) and Magnetic Induction Tomography (MIT), whereas the imaging modality oftenly utilizes Computed Tomography (CT), Magnetic Resonance Imaging (MRI) and Positron Emission Tomography (PET) methods. More recently, the Electromagnetic based Impedance Tomography (EMIT) using microwave signals has been developed as well. In order to make a decision on the selection of an imaging technique for this paper to study stroke detection, a detailed comparative analysis was performed. The comparison highlighted that there is a need for an alternate brain imaging technique which should be low-cost, portable and fast diagnostics modality with safe, non-ionizing and non-invasive features. Therefore, Electromagnetic based Impedance Tomography employing microwave imaging technique is proposed in this study to detect presence and progression of brain stroke.

Microwave imaging (MWI) has been widely used in many industrial and medical applications. The scattered or reflected signals from the target are measured and processed to construct the object images. The operating principle of MWI systems relies on the considerable contrast in the dielectric properties of various regions of the imaged object and its surroundings [5]. In medical imaging applications, EMIT relies on the contrast between dielectric properties of the tissues to classify the normal and abnormal regions. Two major approaches considered in the active MWI are; the Confocal Radar Technique (mono-static, bi-static or multi-static) and the Classical Inverse Scattering (Microwave Tomography) [6, 7] Conventionally in the literature, the layout of EMIT-based head imaging system comprises an array of transmitter-receiver antennas that surrounds the multilayer head and operates in a sequential mode (fig. 1). Microwave signals ( 0.5 to $4.5 \mathrm{GHz}$, power level $\geq 10 \mathrm{dBm}$ ) offer a reasonable compromise between signal penetration into the head and the acceptable spatial resolution of the brain images [8].

The development of EMIT-based head imaging system starts with the evaluation of brain tissues under the direct exposure of microwave signals. Such analysis may be performed in a simulation environment using a realistic human head model, reflecting the heterogenic and anisotropic behavior of head tissues. Mostly, the realistic head models are developed using MRI images or CT data sets. The dielectric properties to different head tissues are assigned using averaged values or frequency-dispersive models [9-13]. To mimic the directional behavior of dielectric properties of brain tissues; diffusion tensor imaging (DTI) or diffusion spectrum imaging (DSI) based mapping is incorporated in the head models [14]. One of the most frequently utilized head models in forward problem modeling is proposed by Zubal, et al. [15]. It is based on 
MRI images and freely accessible [16]. The model has been effectively utilized in several studies conducted by different researchers [7, 17-21].

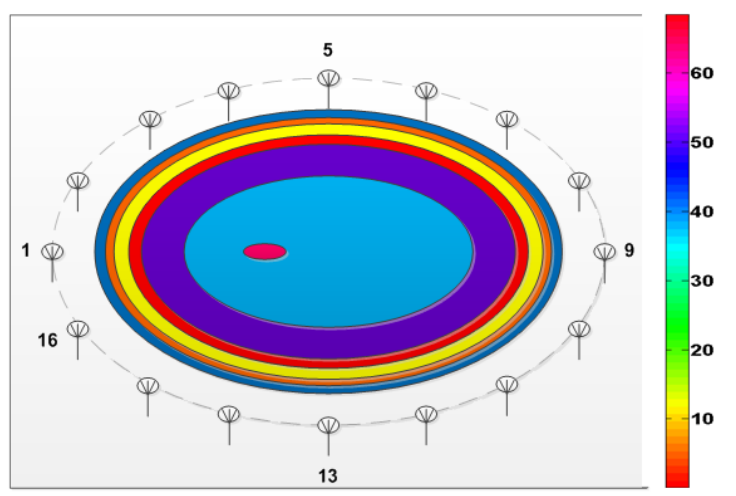

Fig. 1: Layout of an EMIT-based head imaging system

In order to address forward problem modeling, one should take into account the dielectric properties of head tissues. These properties are highly dispersive in the microwave frequency band [22]. K. S. Cole and R. H. Cole provided empirical formula to calculate dispersion and absorption properties of a number of liquids and dielectrics across the wide frequency band, termed as N-term Cole-Cole model [23]. Later on, S. Gabriel and C. Gabriel modified the $\mathrm{N}$-term Cole-Cole model to adapt for assignment of dielectric properties to over 30 body tissues [9-13]. It is termed as 4-term Cole-Cole model and is valid across the frequency range of $10 \mathrm{~Hz}$ to $20 \mathrm{GHz}$. Another less accurate but computationally faster model to calculate complex dielectric permittivity of materials as a function of frequency is known as $\mathrm{N}$-term Debye model. The $\mathrm{N}$-term Debye model was optimized by Ireland and Abbosh [19], to 2-term Debye model to calculate the dielectric properties specifically for head tissues in the microwave frequency band $(0.1$ to $5 \mathrm{GHz})$. Extending Debye's work model Mustafa, et al. [21], modeled human head tissues using 4-term Debye. The model provided an accurate estimation of the dispersive properties of 17 different types of head tissues across the microwave frequency band of 0.1 to $3 \mathrm{GHz}$. The results were more accurate with low computational overheads when compared with 4-term Cole-Cole model.

\section{Background}

The pioneering research in the field of microwave imaging of brain dates back to 1982 when Lin and Clarke [24], experimentally tested the detection of cerebral edema using a $2.4 \mathrm{GHz}$ signal through a rudimentary head phantom. In 2000, this concept was further enhanced by Haddad, et al. [25], to detect hematoma in human brain using ultra-wideband signals. Their work was extended in 2005 by Paulson, et al. [26], but that was of preliminary nature. In 2007 Semenov and Corfield [27], assessed the feasibility and performance potential of Microwave Tomography (MWT) for brain imaging to detect stroke progression, using computer simulations and a simplified 2-D head model with an ischemic stroke.

In 2010 Zakaria, et al. [28], demonstrated the capability of FEM-CSI to detect hemorrhagic stroke inside a simplified 2-D brain model. The data was collected using 32 evenly spaced transmitters in a multi-static radar approach at $1 \mathrm{GHz}$ frequency. In 2011 Ireland and Bialkowski [17], proposed an image reconstruction algorithm based on common background reflections cancellation, confocal delayand-sum approach and Fermat's principle. Its efficacy was tested using a Gaussian pulse $(0.5-2.0 \mathrm{GHz}$ at $20 \mathrm{~dB}$ SNR), Zubal head phantom and the 2-D finite-difference-time-domain (FDTD) method.

In 2012 Scapaticci, et al. [18], performed a feasibility study on microwave imaging for brain stroke monitoring. Firstly, the design guidelines were provided to select the most convenient working frequency $(0.6-1.5 \mathrm{GHz})$ along with optimum matching medium dielectric properties $\left(\epsilon_{\mathrm{mm}}=40, \sigma_{\mathrm{mm}}=0.01 \mathrm{~S} / \mathrm{m}\right)$. These analyses were carried out using a simplified head model based on Transmission Line (TL) theory and single-pole Cole-Cole equation. Secondly, an imaging strategy based on modified Linear Sampling Method (LSM) was also proposed. In 2013, an analytical analysis of stroke detection using ultra-wide band (UWB) radar approach was presented by Jalilvand, et al. [6]. Reflection and transmission scenarios were considered to explain the UWB signals propagation in the human head.

In 2013, a microwave-helmet based measurement setup for the detection and diagnostics of stroke was proposed by Fhager, et al. [29]. This system was aimed at the classification between ischemic and hemorrhagic type strokes. It consisted of a helmet with 10-12 elements triangular patch antenna array $(0.1-3.0 \mathrm{GHz})$ and water filled flexible plastic bags. In 2013, a wideband microwave system for head imaging, utilizing a semielliptical array of 16 corrugated tapered slot antennas (1-4 GHz) installed on an adjustable platform, was presented by Mohammed, et al. [8], and Abbosh [30]. A preprocessing algorithm was applied to extract the target response from the strong background reflections [31]. A post-processing algorithm based on confocal delay-and-sum approach and Fermat's principle was developed to construct a high-resolution image of the phantom. Using similar system Mohammed, et al. [32], also investigated the possibility to differentiate between two kinds of brain stroke. The proposed method was based on a comparison of reflection coefficients $\left(\mathrm{S}_{11}\right)$ of any pair of antennas $(2.0-3.5 \mathrm{GHz})$, located symmetrically around the head. A similar study was conducted by Mobashsher, et al. [33], but on the basis of comparison of reflection phases using wideband unidirectional antennas (1-3 GHz).

An investigation through Finite Element Analysis (FEA), for the possibility of diagnosing the types of brain stroke using microwave imaging system $(1 \mathrm{GHz})$, was carried out in 2013 by Priyadarshini and Rajkumar [34]. The forward problem was solved using Finite Element Method and the inverse scattering problem was solved using Contrast Source Inversion (CSI) method. In 2014, a portable microwave system to detect the TBIs and the intracranial hemorrhage was presented by Mobashsher and Abbosh [35, 36]. The wideband system utilized a unidirectional antenna (1.1-3.4 $\mathrm{GHz}$ ), a virtual arrayed mono-static radar approach, a preprocessing noise removal technique, a back projection image reconstruction algorithm and a fabricated realistic head phantom with emulated brain injuries. In our proposed research, we will perform microwave imaging analysis using FEM-CSI approach applied on a more anatomically realistic human head model.

\section{MATERIALS AND METHODS}

Primarily, the study will evaluate the proposed research methodology based on simulations using the realistic human head model as presented in fig. 2. We aim to propose an alternate prototype design, using EMIT head imaging technique and validate the concept on a fabricated head phantom or volunteer.

\section{RESULTS AND DISCUSSION}

In this section, we have described the progress made towards the modelling of a realistic human head model and highlighted the properties that need to be incorporated in future. We have also explained the results of our simple forward problem solution along with analytical analysis. Finally, we have proposed the most feasible inversion method to obtain brain images for stroke detection and optimal technical parameters of an EMIT-based head imaging system.

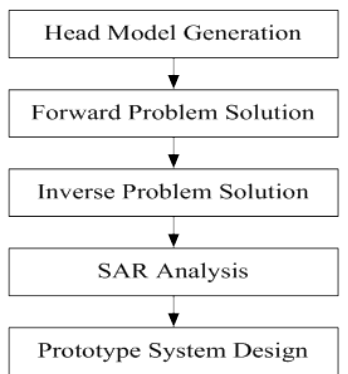

Fig. 2: Proposed research methodology workflow 


\section{Head model generation}

In order to generate a head model, we have run some preliminary simulations using Zubal head phantom MRI data set. The dielectric properties are allocated as it is, to different layers of head tissues using Gabriel database [9-13]. We understand that the verification of microwave systems for head imaging is highly dependent on an anatomically accurate and structurally detailed human head model. The proposed model will, therefore, incorporate the in homogeneous, anisotropic and frequency-dispersive behavior of head tissues' dielectric properties in our future papers. Fig. 3 shows the relative permittivity assignment to different types of head tissues at $1 \mathrm{GHz}$ frequency plotted in MATLAB ${ }^{\circledR}$. We also propose that by using MRI images and DTI or DSI based mapping, the issues of structural details and directional conductivity can be addressed respectively. However, the dielectric properties would be allocated to various types of tissues at microwave frequencies using 4-term Debye expressions.

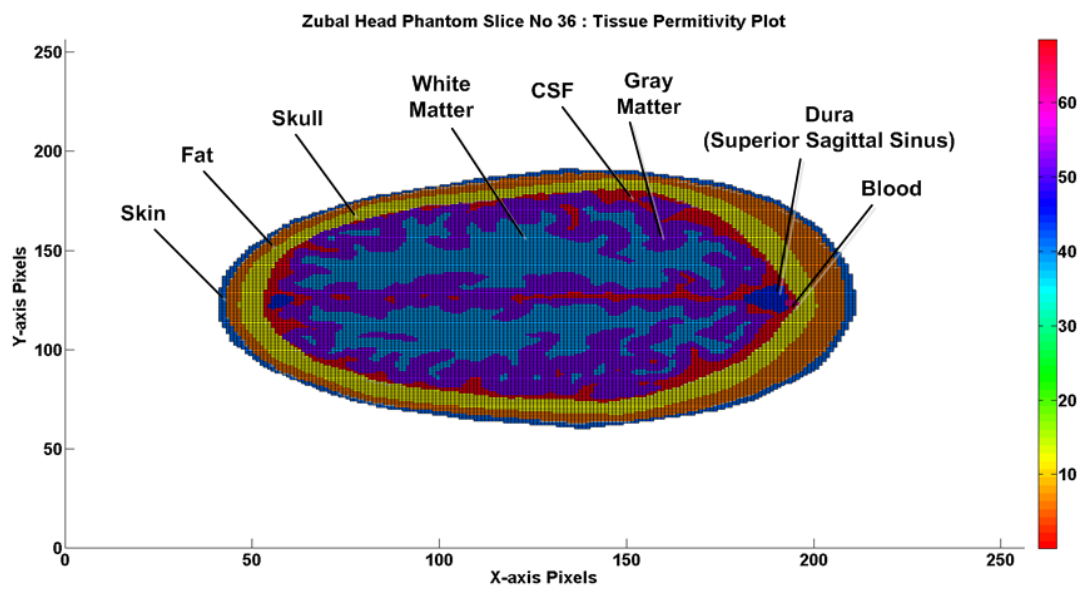

Fig. 3: Relative permittivity of different layers of head tissues at $1 \mathrm{GHz}$

\section{Forward problem solution}

This analysis provides us initial steps to develop a highly anatomically realistic head model at allowable frequency range and the placement of antennas as reported in the literature. Additionally, we propose an inverse problem solution for image reconstruction which is explained in the next section. A simplified 3D ellipsoid head model including hemorrhagic stroke is simulated. A half-wave dipole antenna placed at $4 \mathrm{~cm}$ distance from the side of the head is transmitting a TMpolarized microwave signal at $1 \mathrm{GHz}$ frequency and $13 \mathrm{dBm}$ power. The requisite boundary conditions are also applied in order to realize the real-time electromagnetic interaction phenomena. The spatial distribution of normalized electric field is shown in fig. 4.

In microwave imaging forward problem, the interaction of electromagnetic signals with head tissues is evaluated. The transmitted and backscattered electromagnetic fields at numerous locations are measured by finding the solution of Helmholtz vector wave equation (equation 1), derived from Maxwell's equations in time-harmonic form. Based on the properties of the problem in hand, an appropriate numerical method is selected for solving electric field vector wave equation. Mostly, Finite-Difference Time-Domain (FDTD), Finite Element Method (FEM), Boundary Element Method (BEM) or Method of Moments (MoM) numerical techniques are used in microwave imaging forward solvers [37]. Finite Element Method is adopted, due to its flexibility to deal inhomogeneous background medium, anisotropic dielectric materials and complex geometries with least discretization errors. Therefore, the transmitted and backscattered fields are calculated using Finite Element Method.

$$
\nabla \times \mu_{r}^{-1}(\nabla \times E)-k_{0}^{2}\left(\varepsilon_{r}-\frac{j \sigma}{\omega \varepsilon_{0}}\right) E=0(1)
$$

The analytical analysis of microwave signals interaction with 2-D planar multilayer head model simulated with hemorrhagic stroke is also performed at $1 \mathrm{GHz}$. In this study, a plane wave of $0 \mathrm{dBm}$ power is assumed initially incident at the first interface between the matching material and skin [6]. This may require a transmission power of approximately $8.84 \mathrm{dBm}$ from transmitting antenna placed at $4 \mathrm{~cm}$ distance from the side of the head. In each layer, signal power attenuation due to propagation and the reflection/transmission effects at multiple interfaces are considered using analytical equations. In the present study, only transmission scenario has been investigated and plotted in MATLAB $^{\circledR}$ (fig. 5).

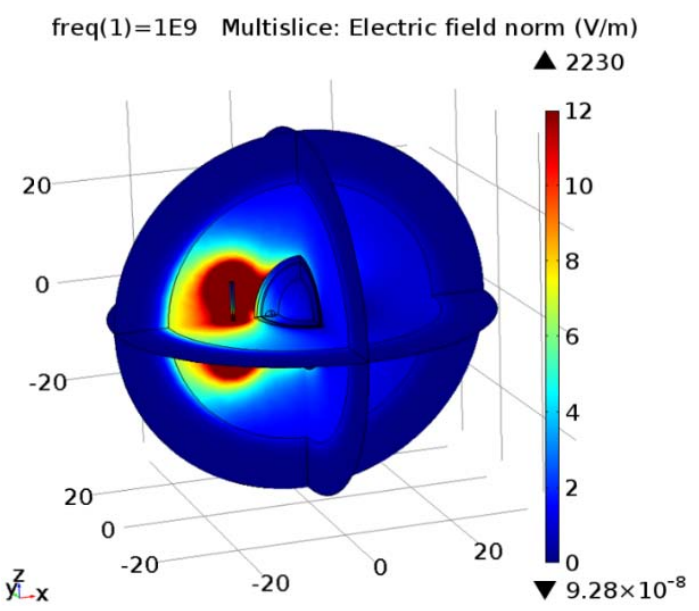

Fig. 4: Spatial distribution of E-Norm in hemorrhagic affected head model at $1 \mathrm{GHz}$

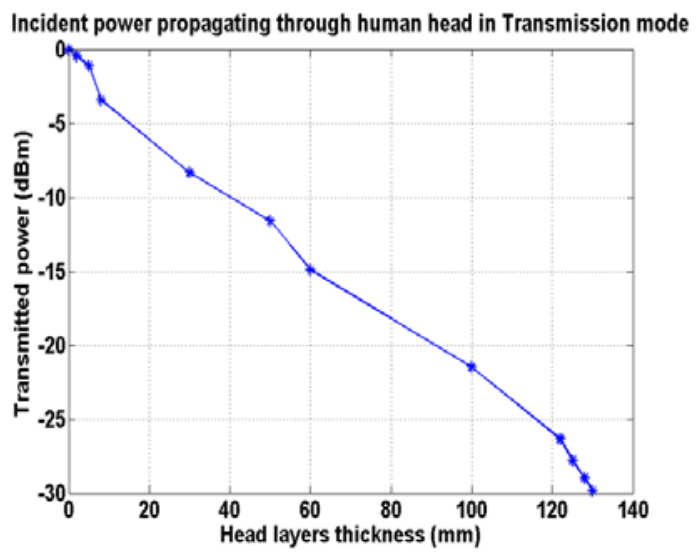

Fig. 5: Incident power propagating through 2-D multilayer head model at $1 \mathrm{GHz}$ 


\section{Inverse problem solution}

In microwave imaging inverse problem, the spatial distribution of the dielectric properties of brain tissues and the shape of the brain are obtained by solving a mathematically nonlinear and ill-posed problem involving a large number of unknowns. The sparse matrices output from the forward solution, containing information on the transmitted and backscattered electromagnetic fields, are fed as an input data to the image reconstruction algorithms. An appropriate image reconstruction method is selected based on the particular application. The most frequently used non-linear inversion methods include; Contrast Source Inversion (CSI), GaussNewton Inversion (GNI), Confocal Delay-and-Sum Algorithm and Born Iterative Method (BIM) [8, 17, 20, 28, 31, 38].

After careful investigation on the comparison analysis of inversion methods suitable for microwave imaging of the head, Contrast Source Inversion (CSI) or Multiplicative Regularized CSI (MR-CSI) is proposed over other methods because it does not require forward solver recalls during multiple iterations [28, 39]. An efficient image reconstruction algorithm based on FEM-CSI will be developed in MATLAB $^{\circledR}$ environment for stroke detection and differentiation. Parallel processing techniques in solving forward and computational inverse problems will also be considered in future studies.

\section{SAR analysis}

The specific absorption rate (SAR) analysis is important because it helps to determine the amount of radiations absorbed by human body tissues and the temperature increase incurred. We investigated only those studies in the literature that involves human head for SAR analysis, mainly using mobile phones [40, 41]. We have also explored that there are certain safety limits to avoid high power and extended exposure of EM waves to the human brain as reported in the literature. Therefore, our proposed research will take into account the position of microwave sources, frequency range, power limits, minimum SNR level and maximum exposure duration. Current studies suggest that a microwave signal (0.5-4.5 $\mathrm{GHz}$ ) with $10-20 \mathrm{dBm}$ power, transmitted at $2-4 \mathrm{~cm}$ distance from the surface of the head phantom, would be sufficient for reconstructing reliable images with minimum ionizing effects to tissues.

\section{Prototype system design}

The outcome of our proposed research will help us to suggest the design of a prototype EMIT-based head imaging system, to verify and validate the concept of microwave imaging for brain stroke detection and classification. Primarily, this will involve the design of a wideband directional antennas array, an experimental chamber to realize the appropriate environmental conditions and an efficient image reconstruction algorithm. Furthermore, necessary hardware setup would also be recommended.

\section{CONCLUSION}

An EMIT-based head imaging system offers the possibility of a lowcost, portable and fast detection imaging modality for brain stroke diagnostics. It can supplement currently available brain imaging and diagnostic tools, due to its safe non-ionizing and non-invasive features. In this paper, we have presented some of the initial results of our proposed research methodology. The inhomogeneous and frequency-dispersive behaviour of human head tissues' dielectric properties has been presented using an anatomically realistic head phantom. The solution of forward model and analytical analysis shows the interaction between electromagnetic signals and a simplified 3-D head model with emulated stroke. At the end, an appropriate inversion technique has been proposed to solve the inverse scattering problem for brain images reconstruction along with EMIT-based head imaging system optimal technical parameters. It is perceived that the development of EMIT-based head imaging system is a challenging task and certain challenges such as; high-contrast and high-loss multi-layered dielectric composition of the human head, design of a wideband directional antennas array, estimation of backscattered fields in a noisy environment and the development of an efficient image reconstruction algorithm, are the major ones.

\section{CONFLICT OF INTERESTS}

\section{Declared none}

\section{REFERENCES}

1. Stroke (Cerebrovascular Accident), Hemorrhagic, Discharge Information. Available from: http://www.summit medicalgroup.com/library/adult_care/ac-

strokehemorrhagic_dc/. [Last accessed on 20 Feb 2016].

2. Feigin VL. Stroke epidemiology in the developing world. Lancet 2005;365:2160-1.

3. The Internet Stroke Center. The Internet Stroke Center, Dallas, TX, USA. Available from: http://www.strokecenter.org. [Last accessed on $20 \mathrm{Feb} 2016$ ]

4. Khan F, Baguley IJ, Cameron ID. 4: rehabilitation after traumatic brain injury. Med J Aust 2003;178:290-5.

5. Pastorino M. Microwave imaging: John Wiley and Sons; 2010. p. 304.

6. Jalilvand M, Li X, Zwick T. editors. A model approach to the analytical analysis of stroke detection using UWB radar. $7^{\text {th }}$ EuCAP Conf: IEEE; 2013.

7. Mohammed BJ, Abbosh A, Bialkowski K, Mustafa S. Investigation of noise effect on image quality in microwave head imaging systems. IET Microw Antennas Propag 2015;9:200-5.

8. Mohammed BJ, Abbosh AM, Mustafa S, Ireland D. Microwave system for head imaging. IEEE Trans Instrum Meas 2014;63:117-23

9. Gabriel C, Gabriel S, Corthout E. The dielectric properties of biological tissues: I. Literature survey. Phys Med Biol 1996;41:2231.

10. Gabriel S, Lau RW, Gabriel C. The dielectric properties of biological tissues: II. Measurements in the frequency range 10 Hz to 20 GHz. Phys Med Biol 1996;41:2251.

11. Gabriel S, Lau R, Gabriel C. The dielectric properties of biological tissues: III. Parametric models for the dielectric spectrum of tissues. Phys Med Biol 1996;41:2271.

12. Gabriel C. Compilation of the dielectric properties of body tissues at RF and Microwave Frequencies. DTIC Document: 1996. p. 16

13. Gabriel C, Peyman A, Grant EH. The electrical conductivity of tissue at frequencies below $1 \mathrm{MHz}$. Phys Med Biol 2009;54:4863-78.

14. Syed Salman S, Marom B, Humaira S, Peng W, Tony A. The value and cost of complexity in predictive modelling: the role of tissue anisotropic conductivity and fibre tracts in neuromodulation. J Neural Eng 2014;11:036002. Doi:10.1088/1741-2560/11/3/036002.

15. Zubal IG, Harrell CR, Smith EO, Rattner Z, Gindi G, Hoffer PB. Computerized three-dimensional segmented human anatomy. Med Phys 1994;21:299-302.

16. Zubal Phantom Data [Online]. Available: http://noodle. med. yale. edu/phantom/getdata. htm.

17. Ireland D, Bialkowski ME. Microwave head is imaging for stroke detection. Prog Electromagn Res M 2011;21:163-75.

18. Scapaticci R, Di Donato L, Catapano I, Crocco L. A feasibility study on microwave imaging for brain stroke monitoring. Prog Electromagn Res B 2012;40:305-24.

19. Ireland D, Abbosh A. Modeling human head at microwave frequencies using optimized Debye models and FDTD method. IEEE Trans Antennas Propag 2013;61:2352-5.

20. Ireland $\mathrm{D}$, Bialkowski $\mathrm{K}$, Abbosh $\mathrm{A}$. Microwave imaging for brain stroke detection using Born iterative method. IET Microw Antennas Propag 2013;7:909-15.

21. Mustafa S, Abbosh AM, Nguyen PT. Modeling human head tissues using fourth-order debye model in convolution-based three-dimensional finite-difference-time-domain. IEEE Trans Antennas Propag 2014;62:1354-61.

22. Dielectric properties of body tissues [Online]; 2002. Available from: http://niremf.ifac.cnr.it/tissprop/htmlclie/htmlclie.php. [Last accessed on $16 \mathrm{Feb} 2016]$. 
23. Cole KS, Cole RH. Dispersion and absorption in dielectrics I. Alternating current characteristics. J Chem Phys 1941;9:341-51.

24. Lin JC, Clarke MJ. Microwave imaging of cerebral edema. Proc IEEE 1982;70:523-4.

25. Haddad W, Chang J, Rosenbury T, Dallum G, Welsh P, Scott D. Microwave hematoma detector for the rapid assessment of head injuries. Lawrence Livermore Nat Lab Tech Rep UCRL-ID; 2000. p. 1379-401.

26. Paulson CN, Chang JT, Romero CE, Watson J, Pearce FJ, Levin N. editors. Ultra-wideband radar methods and techniques of medical sensing and imaging. Optics East: Int Soc Opt Phot; 2005.

27. Semenov SY, Corfield DR. Microwave tomography for brain imaging: feasibility assessment for stroke detection. Int J Antennas Propagation 2008:1-8. Doi.org/10.1155/ 2008/ 254830.

28. Zakaria A, Gilmore C, LoVetri J. Finite-element contrast source inversion method for microwave imaging. Inverse Probl Eng 2010;26:4757-65

29. Fhager A, Yu Y, McKelvey T, Persson M. editors. Stroke diagnostics with a microwave helmet. 7th EuCAP Conf: IEEE; 2013.

30. Abbosh A. editor. Microwave systems for head imaging: challenges and recent developments. IEEE MTT-S IMWS-BIO Workshop; 2013.

31. Mustafa S, Mohammed B, Abbosh A. Novel preprocessing techniques for accurate microwave imaging of human brain. IEEE Antennas Wireless Propag Lett 2013;12:460-3

32. Mohammed B, Abbosh A, Ireland D. editors. Stroke detection based on variations in reflection coefficients of wideband antennas. APSURSI Symp; 2012.

33. Mobashsher AT, Mohammed B, Abbosh A, Mustafa S. editors. Detection and differentiation of brain strokes by comparing the reflection phases with wideband unidirectional antennas. ICEAA Conf; 2013.
34. Priyadarshini N, Rajkumar E. editors. Finite element modeling of scattered electromagnetic waves for stroke analysis. 35th Annu Int Conf EMBC, IEEE; 2013.

35. Mobashsher AT, Abbosh AM, Wang Y. Microwave system to detect traumatic brain injuries using a compact unidirectional antenna and wideband transceiver with verification on the realistic head phantom. IEEE Trans Microwave Theory Technol 2014;62:1826-36.

36. Mobashsher AT, Abbosh A. editors. Microwave imaging system to provide a portable-low-powered medical facility for the detection of intracranial hemorrhage. 1st AMS Symp; 2014.

37. Davidson DB. Computational electromagnetics for RF and microwave engineering. IEEE Aerosp Electro Syst Mag 2005;20:27.

38. Semenov S, Kellam J, Althausen P, Williams T, Abubakar A Bulyshev A. Microwave tomography for functional imaging of extremity soft tissues: a feasibility assessment. Phys Med Biol 2007;52:5705.

39. Zakaria A, Jeffrey I, LoVetri J. Full-vectorial parallel finiteelement contrast source inversion method. Prog Electromagn Res 2013;142:463-83.

40. Morega M, Morega AM. Computed SAR in human head for the assessment of exposure from different phone device antennas. Environ Eng Manage J 2011;10:527-33.

41. Wessapan T, Srisawatdhisukul S, Rattanadecho P. Specific absorption rate a d temperature distributions in human head subjected to mobile phone radiation at different frequencies. Int J Heat Mass Transfer 2012;55:347-59.

\section{How to cite this article}

- Awais Munawar, Zartasha Mustansar, Ahmed E Nadeem, Mahmood Akhtar. An investigation into electromagnetic based impedance tomography using realistic human head model. Int J Pharm Pharm Sci 2016;8 Suppl 2:35-39. 